\title{
Condições ideais para o consumo específico de madeira na produção de celulose
}

\author{
Francisco de Assis Bertini Moraes ${ }^{a}$, Claudio Luis Piratellib*, Jorge Alberto Achcarc \\ afrancisco.bertini@ipaperbr.com, UNIARA, Brasil \\ b*clpiratelli@uniara.com.br, UNIARA, Brasil \\ cachcar@fmrp.usp.br, UNIARA, FMRP/USP, Brasil
}

\begin{abstract}
Resumo
Este artigo apresenta uma modelagem estatística para os dados sobre consumo específico de madeira (CEM) de uma indústria de celulose e papel. Um importante objetivo deste setor é identificar os possíveis fatores que afetam o CEM, promovendo grande variabilidade nos custos de produção. Modelos de regressão linear múltipla foram utilizados para esse fim. Com os fatores identificados, objetivou-se determinar os valores adequados (condições ideais) que minimizam a resposta CEM no processo produtivo através de técnicas de superfície de respostas. Os resultados mostraram que a densidade básica da madeira (DB), o rendimento da madeira no processo (REND) e as perdas na produção (PERDAS) influenciam significativamente o CEM. As condições ideais identificadas foram: DB > $500 \mathrm{~kg}$-seco/m³-sólido; REND > 57\%; PERDAS < 14\%. Conclui-se que, além de trazer contribuições para 0 planejamento de florestas, a abordagem estatística empreendida pode ser útil para o controle dos processos das indústrias de celulose e papel.
\end{abstract}

Palavras-chave

Regressão linear múltipla. Produção de celulose. Análise de superfície de respostas. Consumo específico de madeira.

\section{Introdução}

Segundo Silva (2005), um dos fatores críticos para a competitividade internacional do papel brasileiro é a gestão do processo produtivo. Dentre os diversos fatores que influenciam os custos de produção de celulose e papel, o consumo específico de madeira (CEM) se destaca como um dos mais relevantes - Valverde, Soares e Silva (2006) e Gardner, Little e Arbuthnot (2007). Normalmente, o CEM é expresso em volume $\left(\mathrm{m}^{3}\right)$ de madeira sólida necessária para a produção de uma tonelada de celulose. Dentre as variáveis que afetam CEM estão: a densidade básica da madeira recebida como matéria-prima (DB), o rendimento da madeira nos processos de polpação e branqueamento (REND) e as perdas de madeira que ocorrem no setor de produção de cavacos (PERDAS). Outros fatores, intimamente correlacionados com os anteriores, são: a dilatação ou contração da madeira em função de sua umidade, o teor de casca contida na madeira recebida e a perda de fibras para o efluente no processo de lavagem da polpação e branqueamento - Moraes (2011).

Geralmente as indústrias medem o consumo específico de madeira numa base mensal, em função dos longos tempos de retenção entre a entrada da matéria-prima na área de preparação de madeira e a saída de celulose após o branqueamento. Nessa contabilização mensal estão computados todos os inventários necessários ao processo produtivo. De acordo com Moraes (2011), no Brasil, a variação do CEM nas diversas indústrias instaladas é de $3,55 \mathrm{~m}^{3}$ a $4,50 \mathrm{~m}^{3}$-sólido por tonelada de celulose branqueada produzida. Em 2011, o Brasil produziu um total de 14,1 milhões de toneladas de celulose e de 9,9 milhões de toneladas de papel (ASSOCIAÇÃO..., 2012). Com isso, é fácil perceber a importância econômica de controlar o CEM, por ele influir significativamente nos custos de produção da celulose e evidenciar a necessidade do planejamento de terras e florestas 
para a sustentabilidade da produção em alta escala (BACHMANN, 2009). A busca pela redução do CEM deveria ser enquadrada como uma evidenciação voluntária das práticas ambientais por parte das indústrias - ver Borges, Rosa e Ensslin (2010).

Para o presente artigo foi coletado um conjunto de dados sobre o CEM em uma indústria de celulose localizada no interior do estado de São Paulo, durante o período de janeiro/2004 a junho/2009 - totalizando 66 observações. Associado à resposta CEM foram identificadas as três covariáveis ou fatores previamente apresentados, que poderiam estar relacionados com a variabilidade desses dados: DB, REND e PERDAS. Algumas estatísticas descritivas desses dados são apresentadas na Tabela 1.

Para a análise estatística dos dados da indústria em questão inicialmente procedeu-se a uma transformação da resposta dada pelo inverso do consumo específico de madeira, definida como $Y=1 /$ CEM. Essa transformação da resposta simplifica as interpretações, pois menor CEM significa maior valor da resposta Y e, também, melhora o ajuste do modelo de regressão linear múltipla que será empregado para a análise estatística dos dados. Cabe acrescentar que os interesses dos engenheiros de produção e administradores da indústria objeto de estudo são identificar os fatores importantes que influenciam a variabilidade da resposta CEM e determinar os níveis desses fatores que maximizam Y (condições ideais).

A Figura 1 apresenta os gráficos da resposta $Y$ versus DB, REND e PERDAS.

A partir dos gráficos da Figura 1, algumas hipóteses preliminares relacionando as covariáveis com a resposta Y são apresentadas: aparentemente há uma queda do consumo de madeira (ou seja, $Y$ cresce) com o aumento de DB e o aumento de REND. Para a outra covariável, PERDAS, observa-se um efeito inverso, isto é, $Y$ decresce (aumento do consumo de madeira) quando a covariável PERDAS aumenta. Pode-se observar uma forte relação linear entre a variável resposta e as covariáveis DB, REND e PERDAS, com correlações de Pearson dadas por 0,702 ( $p$-valor $<0,001)$, 0,807 ( $p$-valor $<0,001$ ) e, $-0,494$ ( $p$-valor $<0,001$ ), respectivamente. Os p-valores estimados para o teste de correlação linear mostram correlações significativas ao nível de 5\% de significância.
Apresentado o problema, definiu-se como objetivo central deste artigo verificar o efeito conjunto dessas covariáveis na resposta $\mathrm{Y}$ através de técnicas de regressão múltipla - ver, por exemplo, Draper e Smith (1981), Seber e Lee (2003) ou Montgomery e Runger (2011). Serão utilizadas técnicas de superfície ajustada para explorar o melhor modelo de regressão ajustado por mínimos quadrados para determinar regiões dos níveis das covariáveis (condições ideais) que otimizam $Y$ dentro dos limites de variabilidade de cada covariável - ver, por exemplo, Myers (1971), Box e Draper (1987), Khuri e Cornell (1987) ou Montgomery (2009).

Grande parte dos estudos sobre CEM pertence à área florestal e, em geral, foram conduzidos laboratorialmente, com objetivos distintos dos apresentados acima (ver seção 2.4). Na literatura não foram identificados trabalhos que utilizassem dados reais de produção industrial (com a significativa amostra de 66 meses), considerando também a covariável perdas no processo (tais perdas são inevitáveis, na prática). Ressalta-se como principal contribuição deste trabalho a possibilidade de replicação das técnicas aqui empregadas para a determinação das condições ideais de REND, DB e PERDAS, as quais minimizam o consumo específico de madeira em outros sistemas produtivos reais, com escala industrial. A identificação das condições ideais permite recomendações para o processo produtivo e para o planejamento sustentável de florestas (ver seção 5).

Conforme Miguel (2007), metodologicamente este trabalho pode ser classificado como aplicado, de

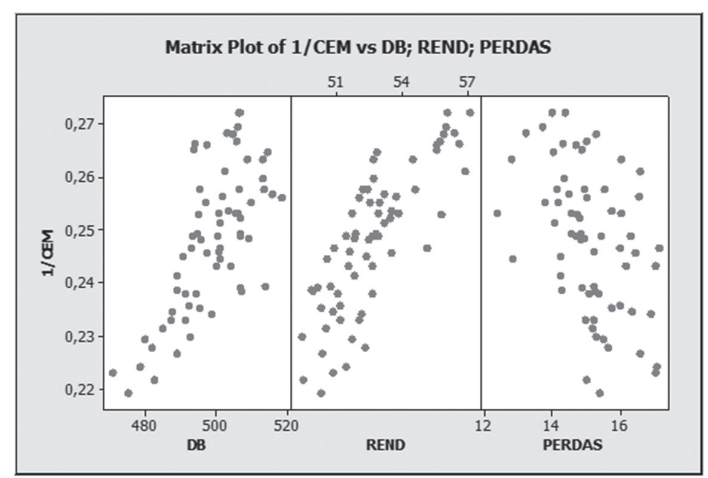

Figura 1. Inverso do consumo específico de madeira versus covariáveis.

Tabela 1. Estatísticas descritivas para os dados da indústria de celulose.

\begin{tabular}{lcccrr}
\hline \multicolumn{1}{c}{ Variável } & Unidade & Média & Desvio padrão & Mínimo & Máximo \\
\hline Consumo específico de madeira (CEM) & $\mathrm{m}^{3} /$ t.celulose & 4,05 & 0,22 & 3,68 & 4,56 \\
Densidade básica (DB) & $\mathrm{kg}$-seco $/ \mathrm{m}^{3}$ & 498,99 & 10,1754 & 471,32 & 518,43 \\
Rendimento da madeira (REND) & $\%$, base seca & 52,66 & 2,00 & 49,40 & 57,11 \\
Perdas de madeira no processo (PERDAS) & $\%$, base seca & 16,08 & 1,66 & 12,11 & 20,59 \\
\hline
\end{tabular}


objetivo descritivo e abordagem quantitativa. Bertrand e Fransoo (2002) definem a pesquisa quantitativa em Engenharia de Produção como aquela em que se modela um problema cujas variáveis apresentam relações causais e quantitativas. Nesse sentido, torna-se possivel quantificar o comportamento das variáveis dependentes sob um domínio específico, permitindo ao pesquisador realizar predições. Em geral, as pesquisas quantitativas utilizam modelagem matemática, estatística ou computacional (simulação) - especificamente, neste trabalho será adotada a modelagem estatística. Quanto às técnicas de pesquisa, serão utilizadas a pesquisa bibliográfica e a observação direta intensiva, segundo a classificação de Lakatos e Marconi (2008), ou a pesquisa bibliográfica e o estudo de caso, conforme a classificação de Gil (2008).

0 artigo é organizado da seguinte forma: a seção 2 apresenta brevemente o processo de produção de celulose, uma breve explanação sobre as covariáveis que afetam o consumo específico de madeira e uma revisão de trabalhos que relacionam CEM, REND e DB; na seção 3 será apresentada a modelagem estatística empreendida para abordar o problema; na seção 4 serão apresentados os resultados obtidos para os dados da indústria de celulose - disponibilizados em Apêndice; na seção 5 será realizada uma discussão dos resultados; e, finalmente, na seção 6 serão tecidas algumas considerações finais.

\section{0 processo de produção de celulose}

De acordo com Magaton Silva et al. (2009), o processo kraft de produção de celulose pode ser considerado uma commodity por ser bem definido e de amplo domínio. De forma sucinta, pode-se dizer que sua principal função é dissolver e extrair a lignina da madeira, com o objetivo de liberar as fibras com o mínimo de degradação dos carboidratos (celulose e hemicelulose). Para maiores detalhes sobre o processo kraft recomenda-se a leitura de Shreve e Brink Junior (1997), Piotto (2003), Colodette et al. (2006), Neuberger (2008) e Moraes (2011).

0 processo, ilustrado pela Figura 2, pode ser dividido em quatro áreas:

1) Linha de fibras;

2) Recuperação química;

3) Utilidades; e

4) Máquina de papel.

A linha de fibras é composta dos processos: preparação da madeira, cozimento, pré-lavagem e depuração, deslignificação com $\mathrm{O}_{2}$, pós-lavagem, branqueamento, secagem e enfardamento da celulose. Essa linha possui ainda uma planta química para produção de dióxido de cloro e armazenagem de peróxido de hidrogênio e soda cáustica, utilizados no processo de branqueamento.

0 processo de recuperação química compõe-se de: evaporação e stripping, caldeira de recuperação, caustificação e forno de cal fornecendo $\mathrm{CO}_{2}$ para produção de carbonato de cálcio destinado à utilização no papel. Engloba também a armazenagem e preparação de enxofre e soda cáustica rayon para reposição das perdas de sódio e enxofre no ciclo de recuperação química.

A área de utilidades subdivide-se em: tratamento de água, tratamento de efluentes, desmineralização de água para as caldeiras, caldeira de biomassa, caldeira a óleo, turbinas e geradores, compressores e distribuição de ar comprimido e distribuição de energia elétrica e vapor.

A área de máquina de papel consome as fibras para confecção do papel utilizando a energia produzida nas áreas de utilidades e recuperação.

Na Figura 3 é possível observar a atuação dos fatores que afetam o consumo específico de madeira no processo de produção de celulose e papel (linha de fibras).

\subsection{Densidade básica da madeira}

Tradicionalmente, na área florestal, a unidade de medida para madeira é dada em volume ( $\mathrm{m}^{3}$-sólido), tanto para quantificação de inventário florestal como para comercialização e transporte. No entanto, a produção de celulose é mensurada em unidade de peso (tsa, tonelada seca ao ar), que demanda quantidade de madeira também em peso ( $t$-seca, tonelada seca). Daí a necessidade de se conhecer a densidade básica da madeira ( $\mathrm{kg}$-seco $/ \mathrm{m}^{3}$-sólido) e verificar sua influência no consumo específico de madeira, em volume. Salienta-se que se o consumo específico de madeira fosse expresso em peso (ou seja, t-madeira seca por tsa de celulose) não seria afetado pela variável DB - exceto pela influência dessa no processo de produção de celulose, ou seja, pelas características da madeira. Para Gomide (2006), o desenvolvimento de florestas com árvores de densidade maior ou igual a $500 \mathrm{~kg}$-seco $/ \mathrm{m}^{3}$-sólido e que apresentam o máximo de rendimento no processo de polpação é a tendência atual de hibridação e clonagem em larga escala de árvores elite.

\subsection{Perdas de madeira no processo industrial}

Além da casca, resíduo indesejável no processo de produção de celulose, há de se considerar perdas de 


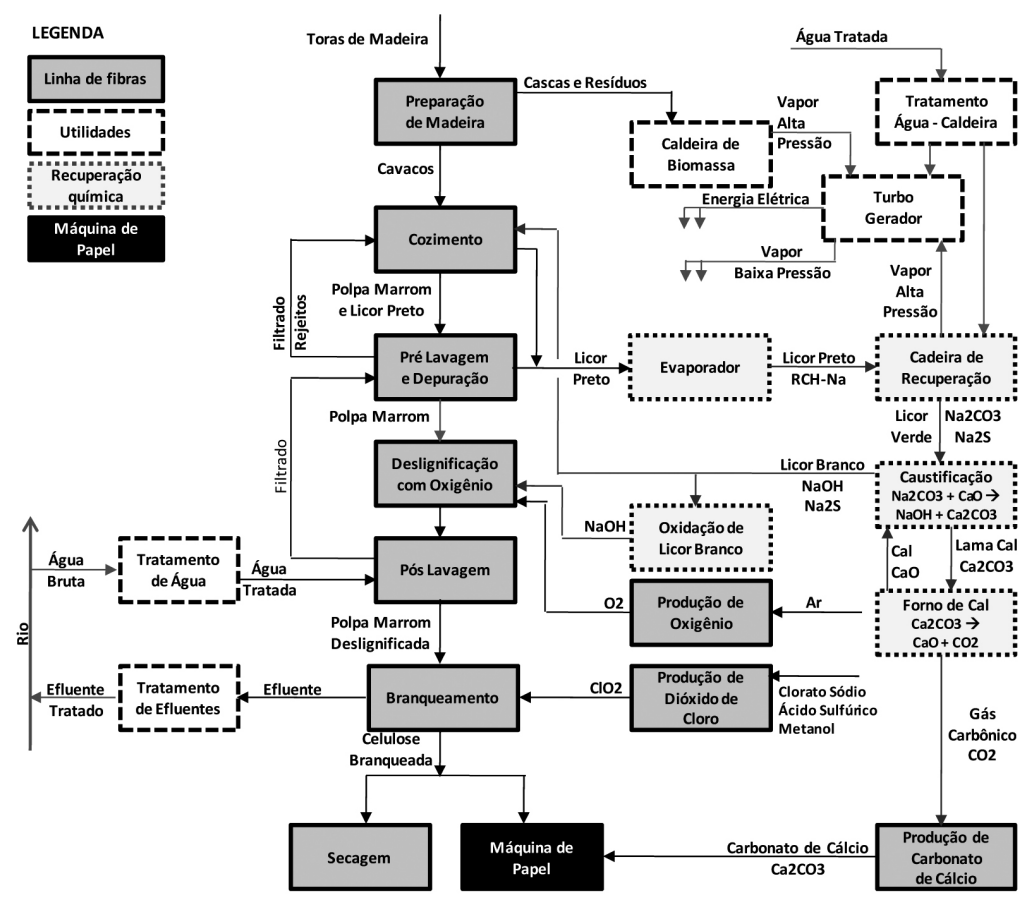

Figura 2. Visão geral do processo kraft de produção de celulose. Fonte: Moraes (2011).

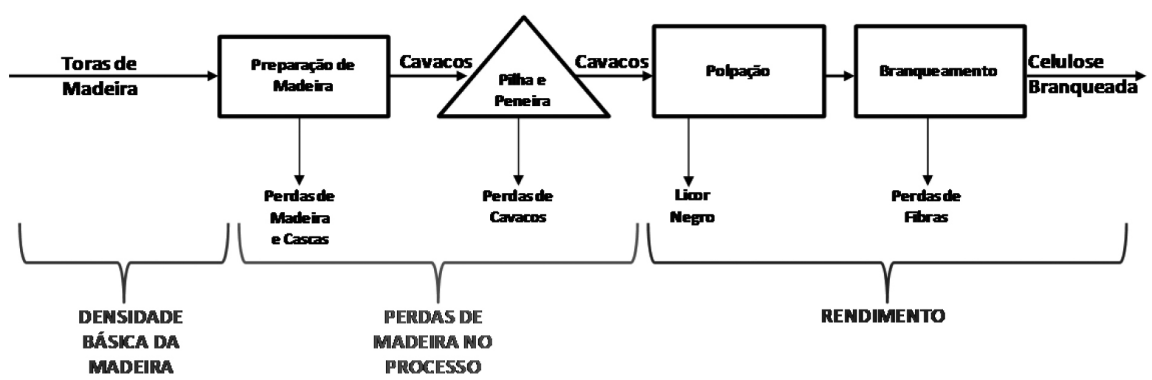

Figura 3. Atuação dos fatores que afetam o consumo específico de madeira no processo produtivo. Fonte: Moraes (2011).

madeira no processo de produção de celulose devido à quebra das toras no tambor descascador, o que gera toretes direcionados como resíduos através de fendas de retirada (de casca do tambor descascador) e das aberturas entre os rolos aceleradores após o tambor descascador. Também ocorrem perdas de madeira na forma de cavacos, principalmente na peneiragem, quando cavacos fora de especificação são rejeitados pela peneira e se incorporam aos resíduos, ou devido ao excesso de alimentação de cavacos para a peneira, o que provoca transbordo de cavacos bons para o rejeito.

Normalmente, a perda de madeira no processo varia de $10 \%$ a $18 \%$ em peso, base seca, do total de madeira com casca consumida, sendo $8 \%$ a $12 \%$ desse total representado pelas cascas, ou seja, as perdas de madeira no seu processamento podem variar de $2 \%$ a 8\%, dependendo de como estão sendo efetuadas as operações em termos de geração de toretes no descascamento e de rejeição de cavacos na peneiragem.

\subsection{Rendimento da madeira}

De um total de $70 \%$ de celulose e hemicelulose contidos na madeira, somente entre $49 \%$ e 56\% serão transformados em polpa branqueada, ou seja, celulose. Isso se deve principalmente à dissolução da celulose e hemicelulose no processo de polpação, onde esses materiais fibrosos se dissolvem juntamente com a lignina, no licor preto destinado à queima do material orgânico (fonte de energia), à recuperação de químicos, na área de recuperação e também, em menor escala, ao rejeito de processo de depuração, 
para retirada de impurezas da celulose branqueada (que representam entre $0,1 \%$ a $0,5 \%$ da produção, dependendo do tipo de equipamento e das condições da manutenção efetuada) - Gomide (2006). De uma maneira geral, quanto maior o rendimento, menor o consumo de madeira para produção de uma tonelada de celulose.

\subsection{Revisão da literatura}

Alguns poucos estudos da área florestal relacionam DB, REND e CEM.

Maron e Neves (2004) analisaram que misturas de outras espécies de madeira com Eucalyptus grandis podem promover um aumento na densidade básica, uma pequena diminuição do rendimento da polpação e uma queda sensível do consumo específico de madeira.

Bassa, Silva Junior e Sacon (2007), a partir de experimentos laboratoriais, afirmam que a mistura de 10\% de Pinus taeda ao Eucalyptus grandis melhora o rendimento da polpação e o consumo específico de madeira.

Gardner, Little e Arbuthnot (2007) compararam a eficiência econômica (função do CEM) de várias espécies de madeira do sul da África na produção de celulose utilizando como covariáveis REND e DB. Em geral, o estudo mostra que quanto maior a DB, maior o REND e menor o CEM.

Mokfienski et al. (2008), a partir de amostras de cavacos de eucalipto, concluíram que menor DB implica em maior REND. Contudo, o CEM é menor para o eucalipto com maior DB.

Guerra et al. (2008) identificaram forte correlação entre o REND e o CEM de clones de Eucalyptus globulus com idade entre 5 e 7 anos plantados em uma mesma área geográfica.

Magaton Silva et al. (2009), a partir de procedimentos experimentais com eucaliptos, concluíram que: (1) o CEM é mais fortemente afetado por DB do que por REND - afirmação corroborada pelo estudo de Demuner (2011); (2) REND não apresenta relação com qualquer outra propriedade da madeira, dentre outras afirmações.

Gomide, Fantuzzi Neto e Regazzi (2010) analisaram 75 amostras de clones de eucalipto e identificaram que o teor de lignina e o de extrativos são os principais critérios de qualidade da madeira que afetam significativamente o rendimento da polpação kraft. Os autores afirmam que a DB não afeta significativamente o REND, mas sim o CEM para produção de celulose.

Sansígolo e Ramos (2011) compararam a qualidade de amostras de eucalipto plantadas em três fazendas distintas e identificaram que a densidade básica possui relação com o REND e com o CEM - maior DB promove um maior REND e um menor CEM.

Observa-se que a maioria dos trabalhos acima são estudos experimentais da área florestal, com variáveis controladas - amostras são coletadas nas florestas e o processo de cozimento/branqueamento é realizado em laboratório para analisarem-se as relações entre DB, REND e CEM. Tais situações não conseguem retratar a escala e a realidade industrial - como, por exemplo, a existência de perdas no processo (PERDAS). Em nenhum dos trabalhos acima observou-se a utilização de técnicas para a determinação dos níveis de REND e DB que minimizassem o CEM (condições ideais). Para se alcançar esse objetivo, o presente trabalho utiliza uma ampla base de dados industriais (66 meses) que, na prática, incluem as perdas ocorridas no processo, as diferentes densidades básicas e rendimentos das matérias-primas provenientes de diversas espécies (incluindo clones) e idades.

\subsection{Modelagem estatística}

Para analisar os dados de consumo específico de madeira na indústria de celulose, utilizou-se um modelo de regressão linear múltiplo considerando as três covariáveis introduzidas na Tabela 1 (ver dados no Apêndice). Assim, considerando-se a resposta $Y$ transformada, supõe-se um modelo de regressão linear múltiplo dado por

$y_{i}=\beta_{0}+\beta_{1}\left(x_{1 i}-\overline{x_{1}}\right)+\beta_{2}\left(x_{2 i}-\overline{x_{2}}\right)+\beta_{3}\left(x_{3 i}-\overline{x_{3}}\right)+\varepsilon_{i}$

onde $\mathrm{i}=1,2, \ldots, 66 ; \varepsilon_{1}$ são erros aleatórios supostos como independentes, com uma distribuição normal com média zero e variância constante $\sigma^{2} ; \mathrm{x}_{1 \mathrm{i}}$ denota a densidade básica (DB) para a i-ésima observação; $\mathrm{x}_{2 \mathrm{i}}$ denota as perdas em madeira (PERDAS) para a i-ésima observação; $\mathrm{x}_{3 \mathrm{i}}$ denota o rendimento de madeira (REND) para a i-ésima observação; $\bar{x}_{l}=\sum_{i=1}^{n} x_{l i} / n, l=1,2,3$ são as médias amostrais para cada covariável considerada. Observar que, usando as variáveis independentes transformadas e centralizadas em sua média, temos essas variáveis com valores centrais iguais a zero; essa transformação é importante para usar técnicas de superfície de respostas, onde obtemos direções do máximo da superfície partindo dos valores centrais das variáveis independentes (ver, por exemplo, MYERS, 1971).

Para a procura do ponto ótimo da superfície ajustada (maior resposta Y, isto é, menor consumo de madeira), utilizam-se técnicas de superfície de respostas.

Considerando-se modelos de primeira ordem, isto é, modelos de regressão incluindo só termos lineares, 
utiliza-se uma técnica exploratória (steepest ascent) para encontrar as regiões da superfície que otimizam a resposta - ver, por exemplo, Myers (1971).

Supor que o modelo ajustado por mínimos quadrados seja dado por

$\hat{y}=b_{0}+\sum_{l=1}^{k} b_{l} z_{l}$

onde $b_{1}, 1=0,1,2, \ldots, k$ são EMQ (estimadores de mínimos quadrados) dos parâmetros de regressão $\beta_{0}$, $\beta_{1}, \ldots, \beta_{k}$. No caso, $k=3$.

Com as covariáveis $z_{\mathrm{li}}$ transformadas e iguais a $\left(x_{l i}-\overline{x_{l}}\right), \mathrm{i}=1, \ldots, \mathrm{n} ; \mathrm{l}=1, \ldots, \mathrm{k}$ o centro da variabilidade das covariáveis é dado por: $z_{1}=0$, $\mathrm{z}_{2}=0, \ldots, \mathrm{z}_{\mathrm{k}}=0$. Para achar os valores de $\mathrm{z}_{1}, \mathrm{z}_{2}, \ldots$, $\mathrm{z}_{\mathrm{k}}$ que maximizam a resposta $b_{0}+\sum_{i=1}^{k} b_{i} z_{i}$ sujeito à restrição $\sum_{i=1}^{k} z_{i}^{2}=R^{2}$ (uma hyperesfera de dimensão $\mathrm{k}$ e raio $\mathrm{R}$ fixado), é apresentada a função a ser maximizada, dada por

$$
Q\left(z_{1}, \ldots, z_{k}\right)=b_{0}+\sum_{l=1}^{k} b_{l} z_{l}-\mu\left(\sum_{l=1}^{k} z_{l}^{2}-R^{2}\right)
$$

onde $\mu$ é um multiplicador de Lagrange, usualmente considerado em problemas de otimização. 0 método dos multiplicadores de Lagrange permite encontrar extremos (máximos e mínimos) de uma função de uma ou mais variáveis suscetíveis a uma ou mais restrições. 0 método dos multiplicadores de Lagrange também garante uma condição necessária para a otimização em problemas de otimização com restrição (ver, por exemplo, BERTSEKAS, 1999).

Observar que é necessário fixar diferentes valores de R (raio da hyperesfera) para determinar o caminho ou direção do máximo da superfície de respostas. Esses valores são obtidos resolvendo-se as equações $\partial Q / \partial z_{j}=b_{j}-2 \mu z_{j}=0$,

$\partial Q / \partial \mu=-\left[\sum_{i=1}^{k} z_{i}^{2}-R^{2}\right]=0$

De 4, encontra-se

$z_{j}=\frac{b_{j}}{2 \mu}$

para $\mathrm{j}=1, \ldots, \mathrm{k}$.
Observar que na prática é mais simples selecionar valores de $\mu$ que correspondam a valores nas variáveis independentes as quais correspondam a aumentos na variável Y (ver, por exemplo, MYERS, 1971). Essa escolha é arbitrária. Daí, esse procedimento continua à procura da direção do máximo (ou mínimo) da superfície ajustada, tomando cuidado para não extrapolar para valores fora dos limites de variação das covariáveis.

\section{Resultados obtidos}

Usando o software Minitab versão $14 \mathrm{e}$ considerando-se o modelo 1 , onde a resposta $Y$ foi multiplicada por $10 \mathrm{mil}$ (um procedimento usado apenas com a finalidade computacional de transformar a variável resposta sem implicações nas interpretações), construiu-se a Tabela 2, contendo os estimadores de mínimos quadrados (EMQ) para os coeficientes de regressão do modelo, os erros padrão (EP) dos estimadores obtidos, a estatística $t$ de Student observada e os valores-p.

Um estimador da variância $\sigma^{2}$ do erro é dado a partir da soma de quadrados residual por $\mathrm{S}^{2}=1.622,49$ - ver, por exemplo, Draper e Smith (1981), Chatterjee, Hadi e Price (1999) -; o coeficiente de determinação que mede a qualidade do ajuste do modelo aos dados é igual à $\mathrm{R}^{2}=0,915$ (um indicativo do excelente ajuste do modelo aos dados). A adequabilidade do modelo (normalidade dos erros, variância constante, erros não correlacionados) também é verificada a partir de gráficos dos resíduos (ver Figura 4).

Dos resultados da Tabela 2 observa-se que as covariáveis densidade básica (DB), perdas de madeira no processo (PERDAS) e rendimento (REND) apresentam efeitos significativos na resposta $Y$, pois os valores- $p$ correspondentes são menores do que 0,05. Esses resultados corroboram as hipóteses preliminares dos dados, apresentadas na seção 1 .

Dos estimadores de mínimos quadrados (EMQ) para os parâmetros de regressão $\beta_{l}, 1=1,2,3$, observa-se que aumentos na DB e no REND ( $\beta_{1}=5,90$ e $\beta_{3}=43,49$ ) levam a um aumento médio na resposta $\mathrm{Y}$, pois os sinais dos estimadores são positivos. Dessa forma, pode-se concluir, há queda no consumo médio

Tabela 2. Estimadores de mínimos quadrados.

\begin{tabular}{lccrr}
\multicolumn{1}{c}{ Preditor } & EMQ para $\beta_{1}$ & EP do estimador & Estatistica t & valor- $\mathrm{p}$ \\
\hline Constante & $-2.404,10$ & 307,60 & $-7,82$ & $0,00\left(^{*}\right)$ \\
Densidade básica (DB) & 5,90 & 0,54 & 10,84 & $0,00\left(^{*}\right)$ \\
Perdas de madeira no processo (PERDAS) & $-23,30$ & 5,10 & $-4,54$ & $0,00\left(^{*}\right)$ \\
Rendimento da madeira (REND) & 43,49 & 2,63 & 16,57 \\
\hline
\end{tabular}




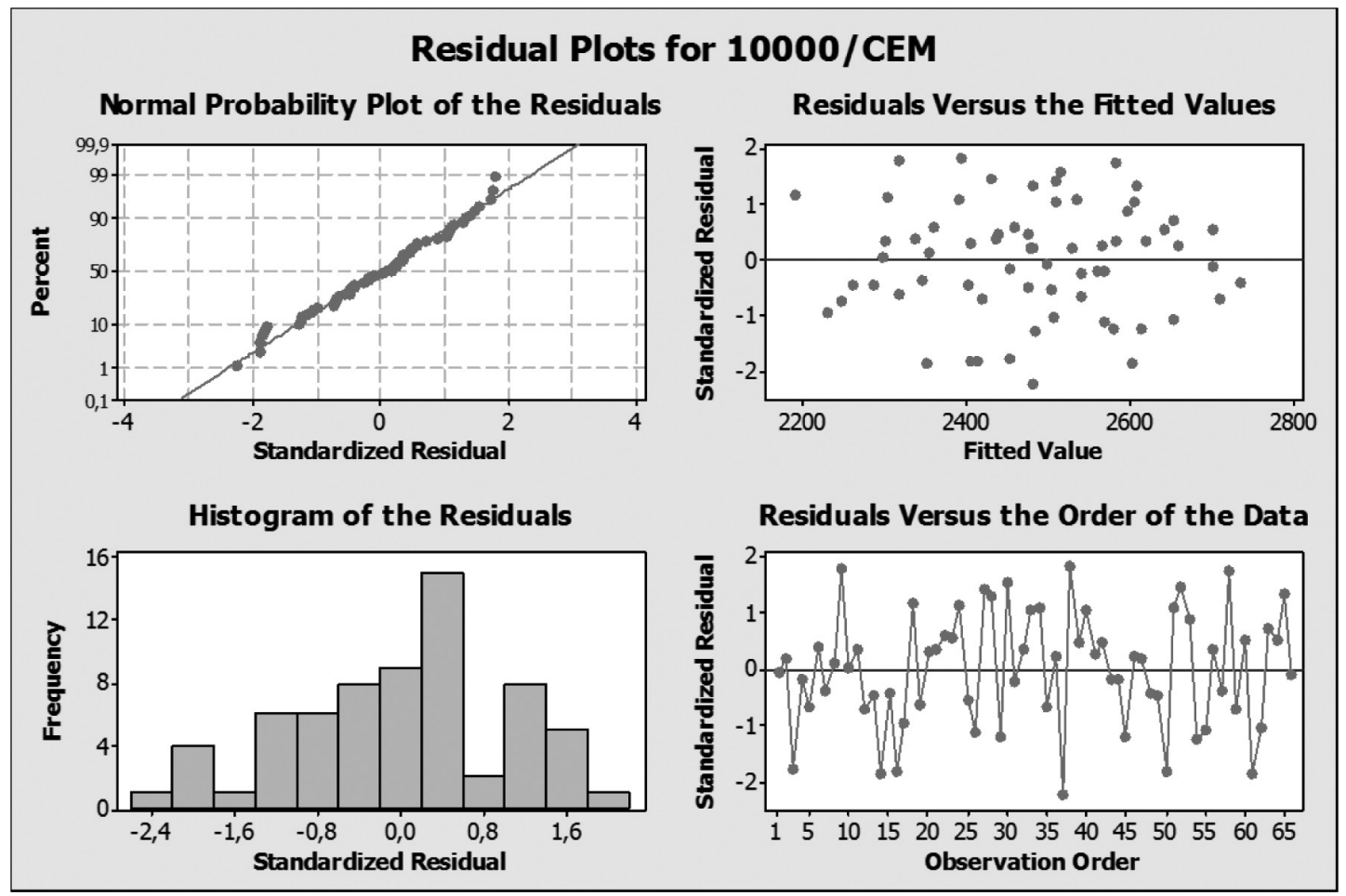

Figura 4. Gráficos dos resíduos padronizados.

de madeira com o aumento dos valores desses fatores (densidade básica e rendimento de madeira). Da mesma forma, um aumento nos níveis de PERDAS leva a um aumento médio no consumo de madeira $\left(\beta_{2}=-23,30\right)$, pois os sinais dos EMQ são negativos.

0 modelo de primeira ordem ajustado é dado por

$(10.000 /$ CEM $)=-2.404,10+5,90(D B)$

- 23,30 (PERDAS) + 43,49 (REND)

onde as covariáveis DB, PERDAS e REND são dadas nas formas transformadas $Z_{1}, Z_{2}$ e $Z_{3}$, respectivamente, com médias iguais a zero, conforme ilustrado nos gráficos da Figura 5.

Com a superfície ajustada dada por 6 é possível fazer previsões para valores especificados das covariáveis $Z_{1}, Z_{2}$ e $Z_{3}$ e encontrar os níveis dos fatores (ou variáveis independentes) que otimizam a resposta $Y$ dentro dos limites observados para as covariáveis: $-27,67 \leq \mathrm{Z}_{1 \mathrm{i}} \leq 19,44 ;-2,706 \leq \mathrm{Z}_{2 \mathrm{i}} \leq 2,022$ e $-3,264 \leq Z_{3 \mathrm{i}} \leq 4,446$.

A partir do centro, onde os valores das covariáveis transformadas são iguais à zero $\left(Z_{1}=0, Z_{2}=0\right.$, $Z_{3}=0$ ), a superfície ajustada é explorada na direção da região do máximo da resposta $Y$.

Para isso, fixam-se valores do raio da hyperesfera de dimensão igual a 3 que satisfazem a equação $\sum_{l=1}^{3} z_{l}^{2}=R^{2}$.
Uma alternativa é fixar um aumento em uma unidade em uma das covariáveis. No caso, optou-se por $\mathrm{Z}_{1}$; daí, determinar o valor de $\mu$ (multiplicador de Lagrange em 3, dado pela Equação $5, \mu=b_{j}$ l $\left(2 z_{\mathrm{j}}\right), \mathrm{j}=1,2,3$.

Assim, com $Z_{1}=0,1$, encontrou-se $\mu=5,9017 /$ $[2(0,1)]=29,5085$ onde $b_{1}=5,9017$ é o EMQ de $\beta_{1}$ (ver Tabela 2). Com esse valor de $\mu$, determinaram-se valores das covariáveis $Z_{2}$ e $Z_{3}$ na direção dos valores maiores da resposta $Y$ obtidos a partir da Equação 5, ou seja,

$Z_{j}=\frac{b_{j}}{2 \mu}$

onde $\mathrm{j}=2,3$ e $b_{\mathrm{j}}$ é o EMQ de $\beta_{\mathrm{j}}$ dado na Tabela 2 . Assim, $Z_{2}=b_{2} / 2 \mu=-23,301 /[2(29,5085)]=-0,394818$. Da mesma forma foi determinado $Z_{3}=43,491 /$ $[2(29,5085)]=0,736923$.

Observar que, em geral, no estudo da superfície de respostas seria necessário fazer novos experimentos com os valores das covariáveis encontrados na direção do máximo da superfície, no caso com os níveis $Z_{1}=0,1, Z_{2}=-0,394818$ e $Z_{3}=0,736923$ - ver, por exemplo, Box, Hunter e Hunter (1978). Como no caso não se dispõe de um estudo experimental, utilizou-se o modelo ajustado 6 para encontrar um valor de previsão para a resposta. 

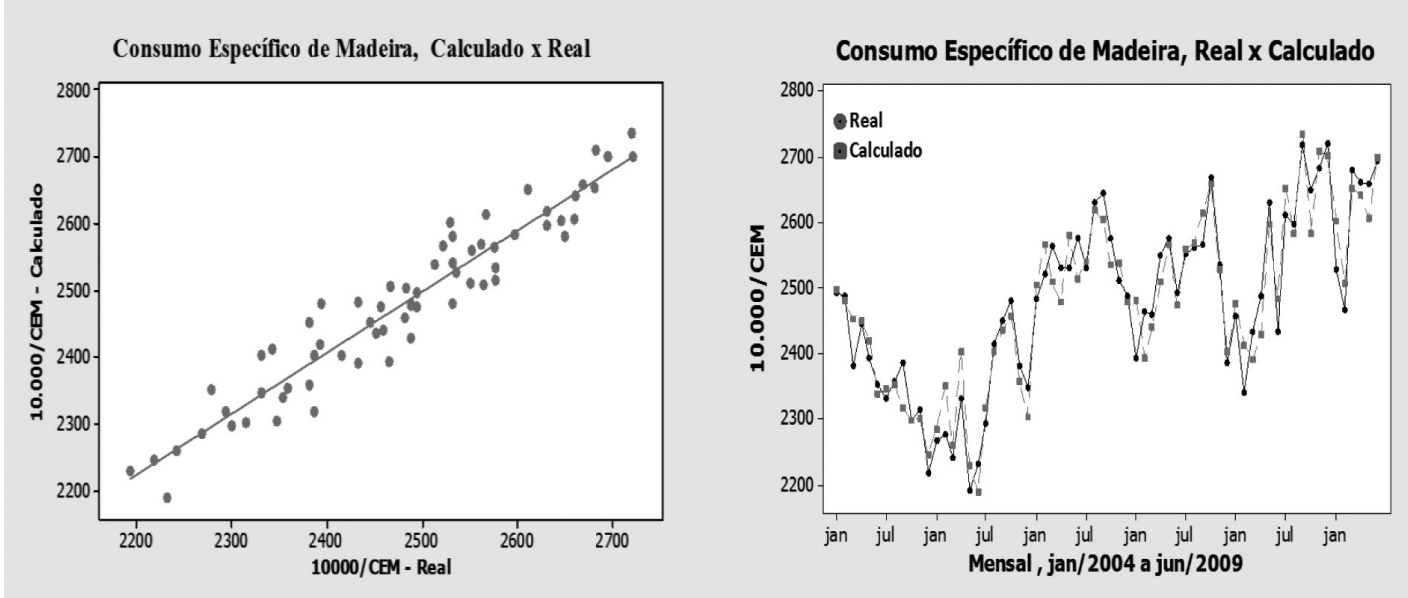

Figura 5. Modelo de primeira ordem ajustado.

Assim, considerando como base inicial para o estudo exploratório as condições (centro da hyperesfera de dimensão 3) $Z_{1}=0, Z_{2}=0$ e $Z_{3}=0$, caminhou-se na superfície ajustada na direção do máximo, tomando cuidado para não extrapolar os valores da variabilidade das covariáveis.

Refazendo o mesmo procedimento considerando $Z_{1}=0,2$, encontraram-se os valores $\mu=14,7543$, $Z_{2}=-0,789634$ e $Z_{3}=1,47384$ na direção do máximo da superfície ajustada. Considerando $Z_{1}=0,3$, encontrou-se $\mu=9,83617, Z_{2}=-1,18445$ e $Z_{3}=2,21077$; com $Z_{1}=0,4$, encontrou-se $\mu=7,37713, Z_{2}=-1,57927$ e $Z_{3}=2,94769$; com $Z_{1}=0,5$, encontrou-se $\mu=5,90170, Z_{2}=-1,97409$ e $Z_{3}=3,68462$; com $Z_{1}=0,6$, encontrou-se $\mu=4,91808, Z_{2}=-2,36891$ e $Z_{3}=4,42154$; com $Z_{1}=0,7$, encontrou-se $\mu=4,21550, Z_{2}=-2,76373$ e $Z_{3}=5,15846$.

Observar na Tabela 3 que o procedimento exploratório deve ser interrompido na base $+6 \mathrm{~d}$ para que todas as covariáveis transformadas tenham níveis dentro dos limites de variação observados, ou seja, $-27,67 \leq Z_{1 \mathrm{i}} \leq 19,44 ;-2,706 \leq \mathrm{Z}_{2 \mathrm{i}} \leq 2,022$ e $-3,264$ $\leq Z_{3 \mathrm{i}} \leq 4,446$. Isso não ocorre na base $+7 \mathrm{~d}$, onde os níveis das variáveis $Z_{2}(-2,76373)$ e $Z_{3}(5,15846)$ extrapolam os limites $-2,706 \leq Z_{2 \mathrm{i}} \leq 2,022$ e $-3,264$ $\leq \mathrm{Z}_{3 \mathrm{i}} \leq 4,446$.

Assim encerrou-se o procedimento exploratório, ficando-se com os niveis $Z_{1}=0,6, Z_{2}=-2,36891$ e $Z_{3}=4,42154$ com resposta estimada igual à $2.730,07$ (ou inverso de consumo específico de madeira igual a 0,2730$)$.

É importante salientar que novos resultados deveriam ser explorados em torno dos níveis $Z_{1}=0,6$, $Z_{2}=-2,36891$ e $Z_{3}=4,42154$ para explorar possíveis direções que minimizem o consumo específico de madeira (ou maximizar seu inverso).
Tabela 3. Valores das covariáveis na direção do máximo da superfície ajustada (menor consumo de madeira).

\begin{tabular}{lcrcc} 
Valores & $\mathrm{Z}_{1}$ & $\mathrm{Z}_{2}$ & $\mathrm{Z}_{3}$ & Resposta 10.000/CEM \\
\hline Base & 0,0000 & 0,0000 & 0,0000 & $2.404,10$ \\
$\mathrm{~d}$ & 0,1000 & $-0,3948$ & 0,7364 & \\
Base +d & 0,1000 & $-0,3948$ & 0,7364 & $2.520,85$ \\
Base +2d & 0,2000 & $-0,7896$ & 1,4738 & $2.562,69$ \\
Base +3d & 0,3000 & $-1,1845$ & 2,2108 & $2.604,54$ \\
Base +4d & 0,4000 & $-1,5793$ & 2,9478 & $2.646,38$ \\
Base +5d & 0,5000 & $-1,9741$ & 3,6846 & $2.688,23$ \\
Base +6d & 0,6000 & $-2,3689$ & 4,4215 & $2.730,07$ \\
Base +7d & 0,7000 & $-2,7637$ & 5,1585 & $2.771,92$ \\
\hline
\end{tabular}

De posse dos níveis ideais para as covariáveis $\left(Z_{1}=0,6, Z_{2}=-2,36891\right.$ e $\left.Z_{3}=4,42154\right)$, determinou-se o consumo específico de madeira ideal igual a 3,6630 $\left(\mathrm{m}^{3} / \mathrm{t}\right.$.Cel $)$ - a partir de $6-$ dado por $\hat{y} \times 10.000=2.730,07$ (ou $\hat{y}=0,2730$ ), que corresponde aos valores na escala original $X_{1}=499,589$ (densidade básica), $X_{2}=12,7321$ (perdas de madeira no processo), $X_{3}=57,0854$ (rendimento da madeira).

\section{Discussão dos resultados obtidos}

A Tabela 4 ilustra as diferenças em custo de dispêndio de madeira e área florestal necessária para manter uma produção anual de 1 milhão de toneladas de celulose. Comparando-se o caso 1 de alta eficiência com o caso 2 de baixa eficiência, encontram-se diferenças de $+28 \%$ no dispêndio de custo de madeira e de $+70 \%$ na área necessária.

Face à grande influência do consumo específico de madeira no custo variável de produção de celulose, o adequado controle dos fatores que o afetam contribui de forma significativa no bom desempenho econômico do negócio. A modelagem matemática, através da 
Tabela 4. Quadro comparativo de consumo de madeira.

\begin{tabular}{cccccccc}
\hline Caso & $\begin{array}{c}\text { CEM } \\
\left(\mathrm{m}^{3} / \mathrm{t} . \mathrm{Cel}\right)\end{array}$ & $\begin{array}{c}\text { Preço madeira } \\
\left(\mathrm{R} \$ / \mathrm{m}^{3}\right)\end{array}$ & $\begin{array}{c}\text { Custo } \\
\text { especifico } \\
(\mathrm{R} \$ / \mathrm{t} . C \mathrm{Cl})\end{array}$ & $\begin{array}{c}\text { Produção } \\
\text { celulose } \\
(\mathrm{t} . \text {.Cel/ano) }\end{array}$ & $\begin{array}{c}\text { Dispendio de } \\
\text { madeira } \\
(\mathrm{MR} \$ / \mathrm{ano})\end{array}$ & $\begin{array}{c}\text { Produtividade } \\
\text { florestal } \\
\left(\mathrm{m}^{3} / \mathrm{ha} . a n o\right)\end{array}$ & $\begin{array}{c}\text { Área florestal } \\
\text { (ha) }\end{array}$ \\
\hline 1 & 3,5 & 60 & 210 & 1.000 .000 & 210 & 40 & 87.500 \\
2 & 4,5 & 60 & 270 & 1.000 .000 & 270 & 30 & 150.000 \\
\hline
\end{tabular}

Fonte: Moraes (2011).

utilização da técnica estatística de regressão linear múltipla, auxilia na determinação dos pesos desses fatores, visando decisões para ações de controle nos valores ótimos, para o mínimo de consumo específico de madeira. A Figura 6 ilustra, em gráficos de superfície, a influência desses fatores no CEM.

Dessa forma pode-se argumentar que para minimizar o consumo específico de madeira (menor que 3,67 $\mathrm{m}^{3} / \mathrm{t}$-celulose), é possível propor as seguintes ações:

- Corroborando Gomide (2006), o desenvolvimento de florestas com árvores de densidade básica maior que $500 \mathrm{~kg}$-seco $/ \mathrm{m}^{3}$-sólido e que apresentem o máximo de rendimento (maior que 57\%) no processo de polpação através de um direcionamento da pesquisa e desenvolvimento para a hibridação e clonagem em larga escala de árvores elite de eucalipto;

- Pesquisa e desenvolvimento direcionados à obtenção de um rendimento da madeira maior que 57\% no processo de produção de celulose, através do aprimoramento e desenvolvimento de novas tecnologias - ver, por exemplo, Bassa, Silva Junior e Sacon (2007), Moraes (2011) e Segura (2012) -, como o cozimento Lo solids ${ }^{\circledR}$ da tecnologia Andritz ou o compact cooking de tecnologia Metso, duplo estágio de deslignificação com oxigênio e branqueamento ECF (celulose sem o uso de cloro elementar), assim como a utilização de aditivos no processo de cozimento, como antraquinona, surfactantes e polissulfetos;

- Maior controle das perdas de madeira no processo - em muitos casos, é onde se encontra o maior potencial de redução no consumo específico de madeira, principalmente devido à falta de medição e controle dessas perdas. Algumas ações são altamente recomendadas na área de preparação de madeira, visando obter uma perda de madeira menor que 13\%:

- Identificar as principais causas de geração de toretes (quebras de toras) no tambor descascador como, por exemplo, controle do tempo após corte da madeira na floresta (teor de umidade da madeira), comprimento ideal da tora em relação às dimensões do tambor descascador, nivel adequado de toras na operação do tambor descascador, rotação adequada do tambor descascador e controle do desgaste da abertura das fendas do tambor descascador, mínimo de distância da
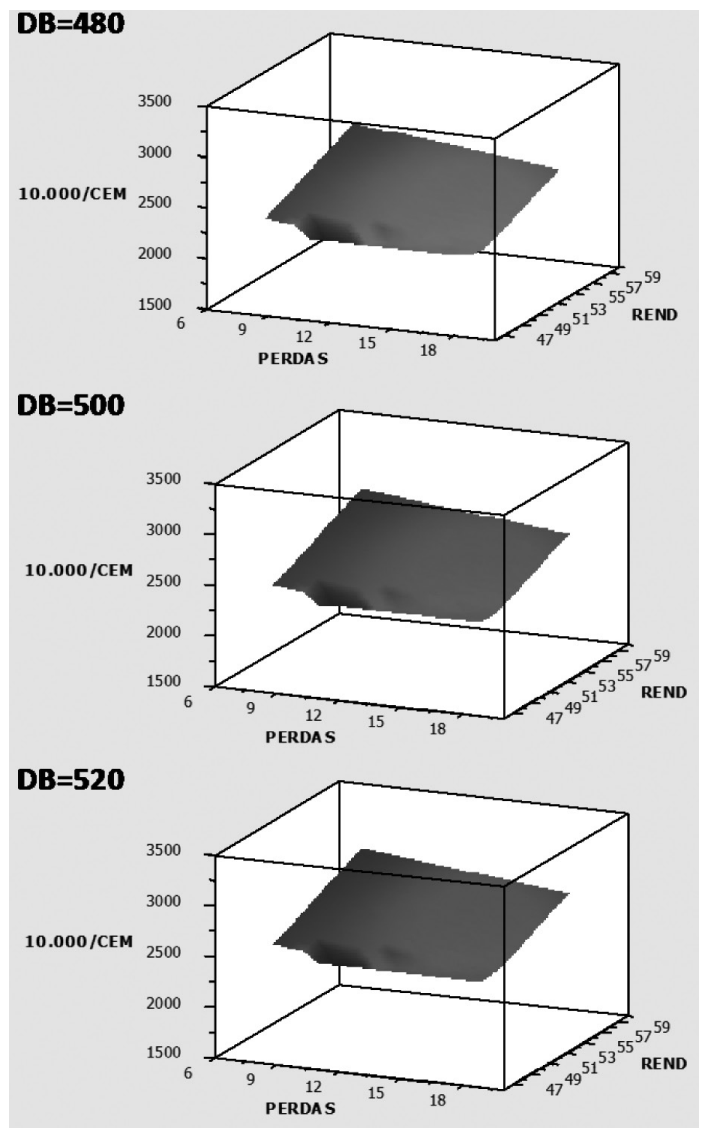

Figura 6. Consumo específico de madeira X Fatores de influência.

abertura entre rolos aceleradores e, se possivel, instalar rolinhos de $50 \mathrm{~mm}$ de diâmetro entre as aberturas maiores;

- Controle adequado do tempo de uso das lâminas do picador, com o objetivo de evitar a formação excessiva de cavacos grandes e de lascas, que certamente serão rejeitados na peneira de cavacos.

Em suma, grandes investimentos são feitos para o desenvolvimento de clones de árvores elite, na aquisição de terras para o reflorestamento e no desenvolvimento de equipamentos e químicos para a obtenção de maior rendimento, não se justificando assim manter altos níveis de perda de madeira no processamento. 


\section{Considerações finais}

Em função da natureza quantitativa e descritiva deste trabalho, optou-se por verificar se a utilização da técnica de modelagem por regressão linear múltipla é uma ferramenta viável, alternativa aos modelos teóricos (com base fenomenológica). A modelagem mostrou-se capaz para abordar um processo de complexidade reconhecida: a interdependência entre as variáveis de influência no resultado econômico da indústria de celulose e papel, relacionadas ao consumo específico de madeira de eucalipto.

Os resultados da regressão linear múltipla dos dados industriais corroboram os efeitos de DB e REND sobre o CEM reportados por Gardner, Little e Arbuthnot (2007), Gomide, Fantuzzi Neto e Regazzi (2010) e Sansígolo e Ramos (2011). Contudo, apresentaram alguma divergência com os resultados obtidos por Mokfienski et al. (2008), Magaton Silva et al. (2009) e Demuner (2011) quanto ao peso de influência de DB e REND sobre o CEM. Salienta-se que as condições experimentais (prática industrial versus experimentos laboratoriais) e as variáveis (espécies de madeira, idades, misturas etc.) utilizadas em cada estudo são distintas, o que sugere novos estudos.

Com o emprego das técnicas de superfície de respostas, foram identificadas as condições ideais para o CEM na indústria objeto de estudo: densidade básica da madeira superior a $500 \mathrm{~kg} / \mathrm{m}^{3}$, rendimento da madeira superior a 57\% e níveis de perda de madeira no processo inferiores a 13\%, garantindo um CEM menor que 3,67 $\mathrm{m}^{3} / \mathrm{t}$-celulose. A determinação dessas condições ideais propiciaram recomendações (apresentadas na seção 5) quanto ao planejamento e ao controle dos processos industriais (processos de polpação e controle das perdas), bem como quanto ao direcionamento de pesquisas para o desenvolvimento de clones de eucaliptos.

As técnicas aqui empreendidas possibilitam a replicação de estudos similares em outras unidades fabris (a partir de dados reais), visando sua eficiência econômica e, consequentemente, a sustentabilidade da cadeia industrial de celulose e papel. Ratifica-se que as condições ideais de CEM aqui reportadas não foram encontradas na literatura industrial.

\section{Referências}

ASSOCIAÇÃO BRASILEIRA DE CELULOSE E PAPEL - BRACELPA. Dados do setor de fevereiro 2012. São Paulo, 2012. Disponível em <http://www.bracelpa. org.br/bra2/sites/default/files/estatisticas/booklet.pdf>. Acesso em: 28 fev. 2012.

BACHMANN, D. Benchmarking dos processos em fábricas de celulose. O Papel, n. 8, p. 66-70, ago. 2009.
BASSA, A. G. M. C.; SILVA JUNIOR, F. G.; SACON, V. M. Misturas de madeira de Eucalyptus grandis x Eucalyptus urophylla e Pinus taeda para produção de celulose Kraft através do Processo Lo-Solids ${ }^{\circledR}$. Scientia Forestalis, n. 75, p. 19-29, set. 2007.

BERTRAND, J. W. M.; FRANSO0, J. C. Operations management research methodologies using quantitative modeling. Journal of Operations \& Production Management, v. 22, n. 2, p. 241-261, 2002. http:// dx.doi.org/10.1108/01443570210414338

BERTSEKAS, D. P. Nonlinear Programming. 2nd ed. Cambridge: Athena Scientific, 1999.

BORGES, A. P.; ROSA, F. S.; ENSSLIN, S. R. Evidenciação voluntária das práticas ambientais: um estudo nas grandes empresas brasileiras de papel e celulose. Produção, v. 20, n. 3, p. 404-417, 2010. http://dx.doi. org/10.1590/S0103-65132010005000034

BOX, G. E. P.; DRAPER, N. R. Empirical model building and response surfaces. New York: Wiley, 1987.

BOX, G. E. P.; HUNTER, W. G.; HUNTER, J. S. Statistics for experimenters: an introduction to design, data analysis and model building. New York: Wiley, 1978.

CHATTERJEE, S.; HADI, A. S.; PRICE, B. Regression analysis by example. New York: Wiley, 1999.

COLODETTE, J. L. et al. Branqueamento de Polpa Kraft de Eucalipto: nível atual da técnica e novos desenvolvimentos. O Papel, v. 67, n. 9, p. 88-110, set. 2006.

DEMUNER, P. W. Predição do Impacto da madeira em fábrica KRAFT de Eucalipto. In: CONGRESSO INTERNACIONAL DE CELULOSE E PAPEL DA ABTCP, 44., 2011, São Paulo. Resumos... São Paulo: ABCTP, 2011. CD-ROM.

DRAPER, N. R.; SMITH, H. Applied regression analysis. Wiley series in probability and mathematical statistics, 1981.

GARDNER, R. A. W.; LITTLE, K. M.; ARBUTHNOT, A. Wood and fibre productivity potential of promising new eucalypt species for coastal Zululand, South Africa. Australian Forestry, v. 70, n. 1, p. 37-47, 2007. http:// dx.doi.org/10.1080/00049158.2007.10676261

GIL, A. C. Como elaborar projetos de pesquisa. 4. ed. São Paulo: Atlas, 2008.

GOMIDE, J. L. Produção de Celulose de Eucalyptus no Brasil: Desafios e Novos Desenvolvimentos. In: CONGRESSO ABTCP/TAPPl, 2006, São Paulo. Anais... São Paulo: SOBER, 2006. CD-ROM.

GOMIDE, J. L.; FANTUZZI NETO, H.; REGAZZI, A. J. Análise de critérios de qualidade da madeira de eucalipto para produção de celulose Kraft. Revista Árvore, v. 34, n. 2, p. 339-344, 2010. http://dx.doi.org/10.1590/S010067622010000200017

GUERRA, A. et al. Influence of Lignin Structural Features on Eucalyptus globulus Kraft Pulping. Industrial \& Engineering Chemistry Research, v. 47, n. 22, p. 85428549, 2008. http://dx.doi.org/10.1021/ie800320d

KHURI, A. 1.; CORNELL, J. A. Response surfaces:designs and analyses. New York: Marcel Dekker, 1987.

LAKATOS, E. M.; MARCONI, M. A. Fundamentos de metodologia científica. 6. ed. São Paulo: Atlas, 2008.

MAGATON SILVA, A. et al. Eucalyptus Wood Quality and lts Impact on Kraft Pulp Production and Use. Tappi Journal, p. 32-40, ago. 2009. 
MARON, A.; NEVES, J. M. Utilização de misturas de cavacos industriais com resíduos de serraria provenientes de madeira de Eucalyptus grandis de diferentes idades para produção de Pasta Kraft. Revista Ciência Florestal, v. 14, n. 1, p. 205-221, 2004.

MIGUEL, P. A. C. Estudo de caso na engenharia de produção: estruturação e recomendações para sua condução Produção, v. 17, n. 1, p. 216-229, 2007. http://dx.doi. org/10.1590/S0103-65132007000100015

MOKFIENSKl, A. et al. A importância relativa da densidade da madeira e do teor de carboidratos no rendimento de polpa e na qualidade do produto. Ciência Florestal, v. 18, n. 3, p. 401-413, 2008.

MONTGOMERY, D. C. Design and analysis of experiments. New York: Wiley, 2009.

MONTGOMERY, D. C.; RUNGER, G. C. Applied statistics and probability for engineers. New York: Wiley, 2011.

MORAES, F. A. B. Modelo para avaliação do consumo específico de madeira e insumos energéticos no processo de produção de celulose e papel. 2011. 212 f. Dissertação (Mestrado em Engenharia de Produção)-Centro Universitário de Araraquara, Araraquara, 2011.

MYERS, R. H. Response surface methodology. Boston: Allyn and Bacon, 1971.

NEUBERGER, R. Boas práticas de operação e manutenção para redução de odores na produção de celulose Kraft: uma abordagem qualitativa. 2008. 125 f. Dissertação (Mestrado em Engenharia de Processos Químicos e
Bioquímicos)-Centro Universitário do Instituto Mauá de Tecnologia, São Caetano do Sul, 2008.

PIOTTO, Z. C. Eco-eficiência na indústria de celulose e papel. 2003. 357 f. Tese (Doutorado em Engenharia)Universidade de São Paulo, São Paulo, 2003.

SANSÍGOLO, C. A.; RAMOS, E. S. Quality of wood and pulp from a clone of Eucalyptus grandis planted at three locations. Cerne, v. 17, n. 1, p. 47-60, 2011.

SEBER, G. A. F.; LEE, A. J. Linear regression analysis. 2nd ed. Wiley series in probability and mathematical statistics, 2003.

SEGURA, T. E. S. Avaliação das Madeiras de Eucalyptus randis vs Eucalyptus urophila e Acacia mearnsii para produção de celulose Kraft pelos Processos convencional e LoSolids ${ }^{\circledR}$. 2012. 99 f. Dissertação (Mestrado em Ciências)Escola Superior de Agricultura "Luiz de Queiróz", Piracicaba, 2012.

SHREVE, R. N.; BRINK JUNIOR, J. A. Indústrias de Processos Químicos. 4. ed. Rio de Janeiro: LTC, 1997. 732 p.

SILVA, C. L. Competitividade internacional da indústria brasileira exportadora de papel de imprimir e escrever à luz da cadeia de valor. Produção, v.15, n. 1, p. 114-126, 2005. http://dx.doi.org/10.1590/S010365132005000100010

VALVERDE, S. R.; SOARES, N. S.; SILVA, M. L. Desempenho das exportações brasileiras de celulose. Revista Árvore, v. 30, n. 6, p.1017-1023, 2006. http://dx.doi. org/10.1590/S0100-67622006000600017

\section{Optimal conditions for wood consumption in cellulose production}

\section{Abstract}

This study presents a statistical modeling approach for wood consumption in the cellulose industry. One of the goals of the cellulose industry is to identify the factors that can affect wood consumption (CEM) and cause high variability in production costs. Multiple linear regression models were used for this purpose. After the factors of influence were identified (for optimal conditions), the goal was to determine their appropriate values to minimize CEM in the production process, using response surface methods. The results showed that three factors affect wood consumption: the basic density (DB) of the wood received, the Kraft pulp yield (REND), and wood losses that occur during the production process (PERDAS). The optimal conditions identified are DB > $500 \mathrm{~kg}$-dry $/ \mathrm{m}^{3}$-solid, REND > 57\% and PERDAS < 13\%. The statistical approach taken in this work is concluded to be useful in process control in the cellulose industry and can also provide insights useful to forest planning.

\section{Keywords}

Multiple linear regression. Cellulose production. Response surface analysis. Pulp wood specific consumption. 


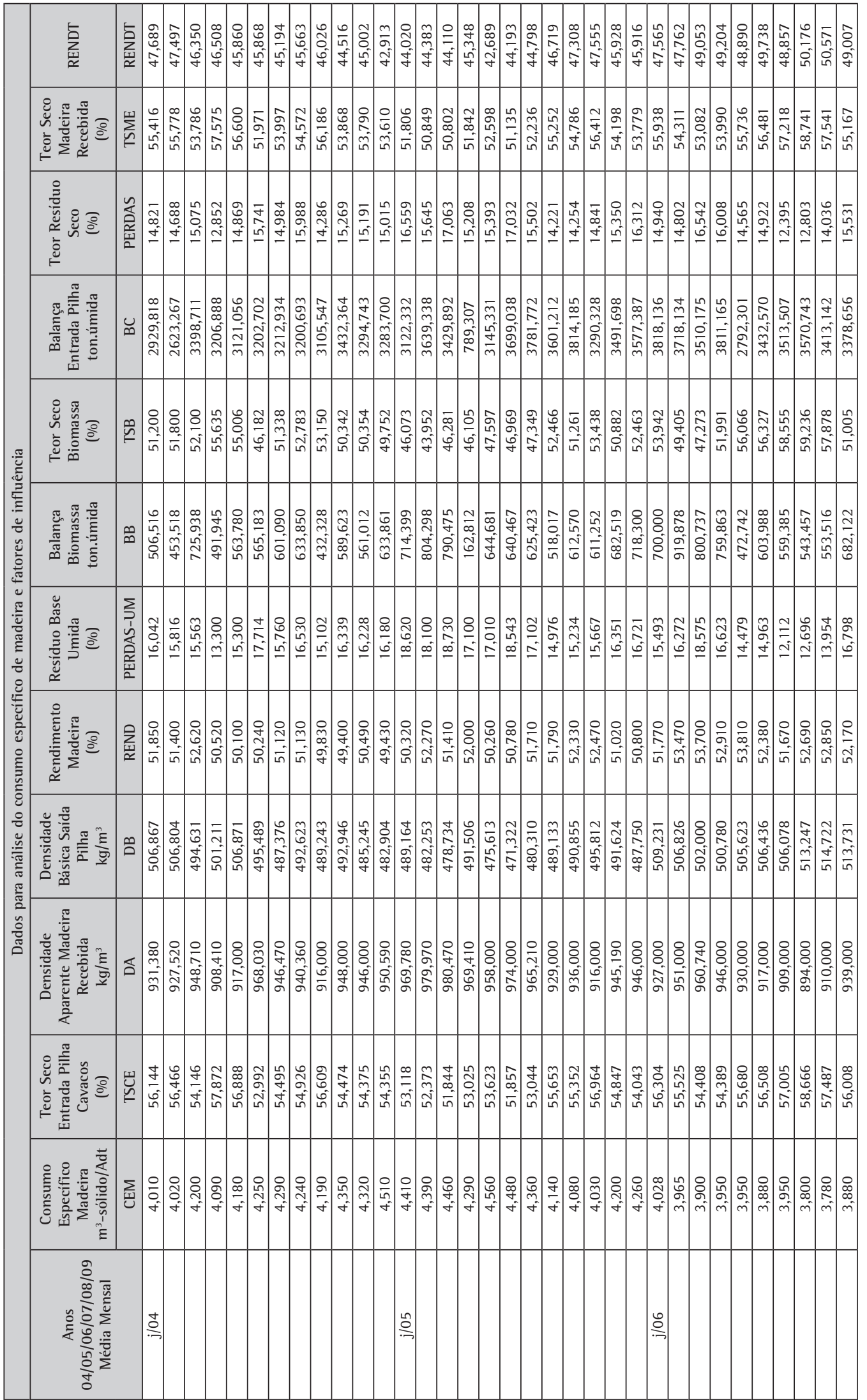




\begin{tabular}{|c|c|c|c|c|c|c|c|c|c|c|c|c|c|c|c|c|c|c|c|c|c|c|c|c|}
\hline & 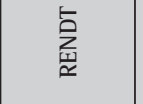 & $\sum_{\mathbb{1}}^{\mathbf{5}}$ & & & $\mid \begin{array}{l}\bar{\sigma} \\
\bar{\sigma} \\
\bar{y} \\
\end{array}$ & & 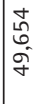 & $\left|\begin{array}{c}\frac{\delta}{2} \\
\frac{\sigma}{\sigma o} \\
\sigma\end{array}\right|$ & 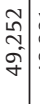 & 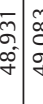 & & & 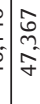 & 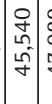 & & & 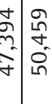 & 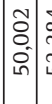 & 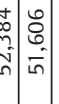 & \begin{tabular}{l|l}
0 \\
$\vdots$ \\
0 \\
$i$ \\
\end{tabular} & 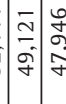 & & & \\
\hline & 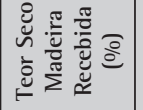 & $\sum_{n}^{m}$ & 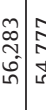 & & $\begin{array}{l}\tilde{0} \\
\hat{n} \\
\hat{n}\end{array}$ & 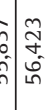 & $\begin{array}{l}0 \\
0 \\
0 \\
0 \\
0 \\
n\end{array}$ & $\frac{\infty}{5}$ & $\begin{array}{c}\tilde{a} \\
\vdots \\
\hat{n}\end{array}$ & เి & $\hat{n}$ & & & $\mid \begin{array}{l}n \\
\sigma \\
\sigma \\
\sigma\end{array}$ & & 章 & 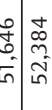 & 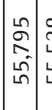 & 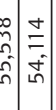 & 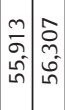 & 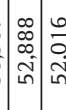 & 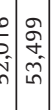 & & \\
\hline & 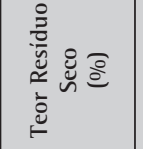 & 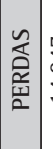 & 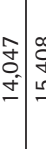 & & 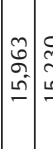 & & $\frac{\mathscr{m}}{\dot{f}}$ & 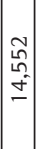 & 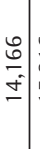 & & $\begin{array}{l}\infty \\
\vdots \\
\vdots \\
\vdots \\
\dot{0}\end{array}$ & & & $\left.\mid \begin{array}{l}\infty \\
0 \\
0 \\
0 \\
- \\
-1\end{array}\right]$ & & $\mid \begin{array}{c}0 \\
0 \\
0 \\
0 \\
0\end{array}$ & 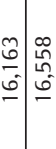 & 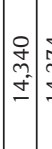 & 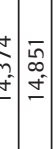 & 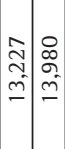 & 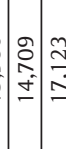 & 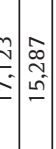 & 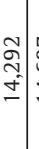 & \\
\hline & 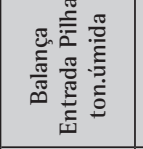 & $\breve{\infty}$ & 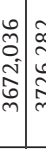 & & 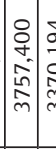 & 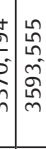 & 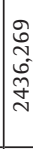 & $\left|\begin{array}{c}n \\
n \\
0 \\
0 \\
0 \\
\tilde{n} \\
n\end{array}\right|$ & 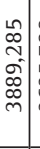 & & 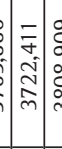 & & חٕ & $\mid \begin{array}{l}0 \\
0 \\
0 \\
\vdots \\
\vdots \\
\vdots \\
\vdots\end{array}$ & & 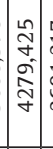 & 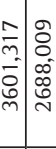 & 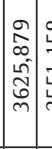 & 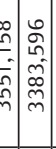 & 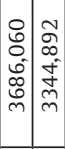 & 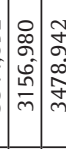 & 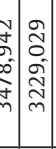 & | & \\
\hline & 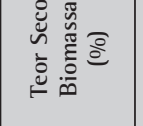 & $\tilde{n}$ & 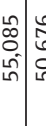 & & & & 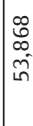 & \begin{tabular}{|c|}
$\infty$ \\
0 \\
0 \\
$\sim$ \\
\end{tabular} & 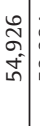 & & $\hat{\vdots}$ & & & $\left|\begin{array}{l}\hat{\Xi} \\
\dot{f} \\
\dot{f}\end{array}\right|$ & & & 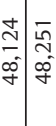 & $\begin{array}{l}0 \\
\tilde{\sigma} \\
\dot{H} \\
\dot{H}\end{array}$ & & 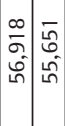 & $\begin{cases}\tilde{n} \\
\hdashline \\
g \\
g\end{cases}$ & 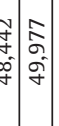 & & \\
\hline 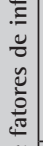 & 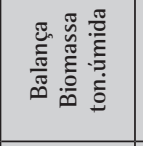 & $\approx$ & ר) & 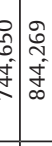 & $\left|\begin{array}{ll}\bar{\sigma} \\
\bar{\infty} \\
\frac{5}{\infty} \\
\bar{\infty}\end{array}\right|$ & $\begin{array}{c}m \\
0 \\
6 \\
6\end{array}$ & $\frac{⿱}{m}$ & 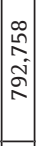 & 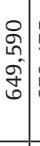 & & 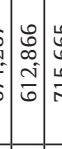 & & $\begin{array}{l}0 \\
\vdots \\
\vdots\end{array}$ & $\mid$ & & & 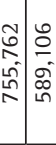 & 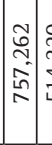 & 年 & 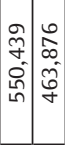 & 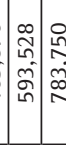 & & & \\
\hline 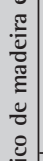 & 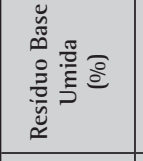 & 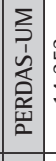 & 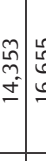 & 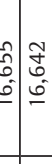 & 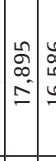 & 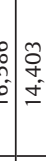 & $\begin{array}{l}\hat{\omega} \\
\infty \\
\dot{ \pm}\end{array}$ & $\frac{\tilde{m}}{\tilde{\omega}}$ & $\begin{array}{l}\frac{v}{m} \\
+ \\
\tilde{f}\end{array}$ & & & & : & $\left|\begin{array}{c}\infty \\
0 \\
\infty \\
\infty \\
0\end{array}\right|$ & & & 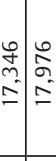 & 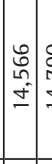 & 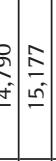 & & 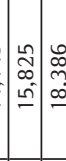 & & & \\
\hline 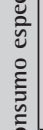 & 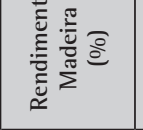 & 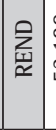 & 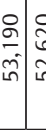 & & & & & $\left|\begin{array}{c}0 \\
0 \\
\hat{i} \\
\tilde{i}\end{array}\right|$ & & & & & & 욘 & & 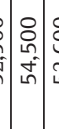 & 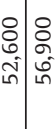 & $\mid \begin{array}{c}8 \\
\vdots \\
i \\
1 \\
i n\end{array}$ & 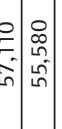 & 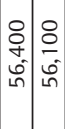 & 量 & 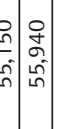 & & \\
\hline 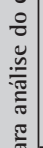 & 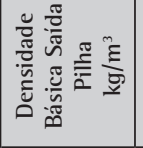 & $\cong$ & 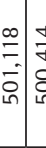 & & & & 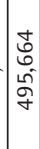 & $\begin{array}{c}0 \\
0 \\
0 \\
\vdots \\
\sigma \\
\sigma\end{array}$ & $\begin{array}{l}9 \\
\vdots \\
0 \\
\text { in }\end{array}$ & 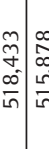 & & & 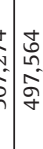 & 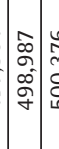 & & $\left\{\begin{array}{l}8 \\
0 \\
0 \\
0 \\
0 \\
0\end{array}\right.$ & 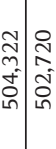 & $\begin{array}{lll}\infty & 0 \\
0 \\
m \\
m \\
\text { in } \\
\vdots\end{array}$ & 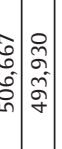 & 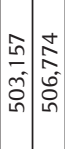 & 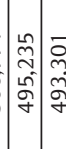 & 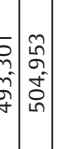 & 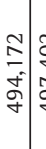 & \\
\hline 0 & 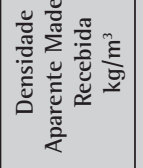 & 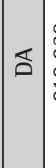 & 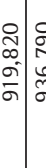 & 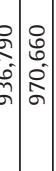 & 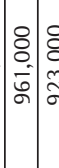 & 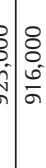 & : & $\begin{array}{l}\vdots \\
\vdots \\
0 \\
0 \\
\sigma\end{array}$ & 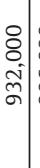 & & $\begin{array}{c}\vdots \\
\vdots \\
\vdots \\
\vdots \\
\vdots \\
\vdots \\
\vdots\end{array}$ & & & & & 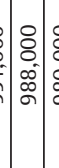 & \begin{tabular}{l|l} 
¿ \\
$\vdots$ \\
$\vdots$ \\
$\vdots$
\end{tabular} & $\begin{array}{l}\delta \\
\delta \\
\sigma^{0} \\
\sigma \\
\sigma \\
\delta\end{array}$ & 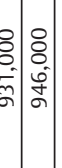 & $\begin{array}{l}8 \\
\text { ठे } \\
\text { ஸे } \\
\text { ڤ్ }\end{array}$ & 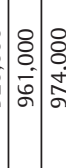 & : & & \\
\hline & 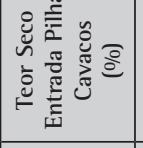 & 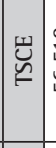 & 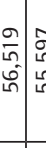 & 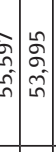 & 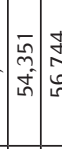 & $\overbrace{}^{\circ}$ & in & 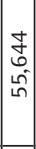 & 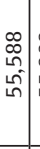 & 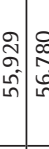 & & & & 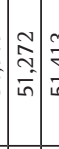 & & 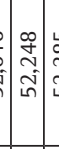 & & {$\left[\begin{array}{c}0 \\
2 \\
0 \\
1 \\
1\end{array}\right]$} & 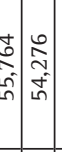 & 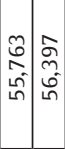 & 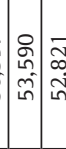 & $\begin{array}{c}n=0 \\
0\end{array}$ & & \\
\hline & 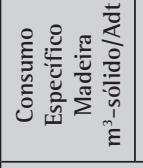 & $\sum_{\text {U. }}$ & & 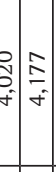 & $\hat{\sigma}$ & & & o. & $\begin{array}{c}\bar{q} \\
\bar{m}\end{array}$ & & $\mid$\begin{tabular}{c}
$\infty$ \\
$\substack{\infty \\
m}$ \\
\hdashline
\end{tabular} & & & 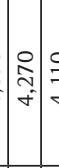 & $\begin{array}{l}0 \\
f \\
f\end{array}$ & $=$ & 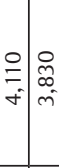 & 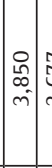 & 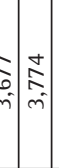 & & 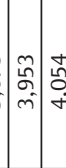 & 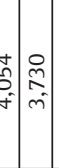 & & \\
\hline & 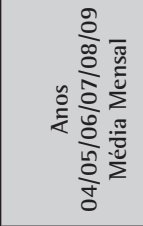 & & & $\stackrel{?}{\rightarrow}$ & & & & & & & & & & & & & & & & & $\stackrel{\circ}{=}$ & & & \\
\hline
\end{tabular}

